\title{
Shapes of epitaxial gold nanocrystals on $\mathrm{SrTiO}_{3}$ substrates
}

\author{
Peiyu Chen, * Krishnan Murugappan, Martin R. Castell*
}

Department of Materials, University of Oxford, Parks Road, Oxford, OX1 3PH, United Kingdom

E-mail: peiyu.chen@materials.ox.ac.uk (P.C.), martin.castell@materials.ox.ac.uk (M.R.C.)

\begin{abstract}
Morphological control of gold nanocrystals is important as their catalytic and optical properties are highly shape dependent. In this paper we report the shapes of gold nanocrystals which deviate from the equilibrium Wulff shape due to the influence of the $\mathrm{SrTiO}_{3}$ single crystal substrates. The gold crystals are characterized by scanning tunneling microscopy (STM) and scanning electron microscopy (SEM). The nanocrystals have an equilibrium shape of a truncated octahedron with $\{111\}$ and $\{001\}$ facets. On all three substrate surfaces, i.e., $\mathrm{SrTiO}_{3}(001)-(2 \times 1), \mathrm{SrTiO}_{3}(001)-c(4 \times 2)$, and $\mathrm{SrTiO}_{3}(111)-(4 \times 4)+(6 \times 6)$, the height-to-width ratio of the gold crystals is not a constant as would be expected for equilibrium crystals, but instead it increases with crystal height. We propose that as the crystals increase in size, their aspect ratio heightens to relax the interfacial strain. The ratio between the $\{111\}$ and $\{001\}$ surface areas of our gold crystals is found to differ on the three substrates, which we speculate is due to the selective adsorption of surfactants on the $\{111\}$ and $\{001\}$ gold facets resulting from the different substrate surfaces. Reentrant facets of gold crystals that should be present according to their Wulff shape are not observed because these concave sites typically grow out due to kinetic considerations. This study demonstrates the significant effect of the crystal facet termination and surface reconstruction of an oxide substrate on the shape of supported gold nanocrystals.
\end{abstract}




\section{Introduction}

In contrast to the inertness of bulk gold, nanosized gold has been a focus of research since the $1980 \mathrm{~s}$ because of its exceptional catalytic activity. ${ }^{1}$ A well-known example is that gold nanoparticles below $5 \mathrm{~nm}$ are a highly efficient catalyst for CO oxidation at room temperature. ${ }^{1-4}$ Their catalytic activity is due to the high fraction of edge/corner atoms, which are the most catalytically active sites. ${ }^{5}$ Different facets of metal nanoparticles also exhibit distinct catalytic properties because of their different atomic arrangements and energies. ${ }^{6,7}$ For example, high-index facets such as $\{730\}$ and $\{210\}$ of $\mathrm{Pt}$ nanocrystals were found to exhibit enhanced catalytic activity (by up to $400 \%$ ) for the electro-oxidation of formic acid and ethanol, which are alternatives for direct fuel cells. ${ }^{6}$ Hence the catalytic activity of gold nanoparticles is a shape-dependent property. In addition, nanogold exhibits interesting optical behavior and it has been extensively studied for its application in surface plasmon resonance (SPR) sensors. ${ }^{8-11}$ Again, the optical properties of gold nanostructures are highly dependent on morphological factors such as size and shape. ${ }^{11-16}$ For example, gold nanocubes, tetrahedra, icosahedra, decahedra, octahedra, and nanospheres all display different SPRs because they possess different proportions of sharp edges. ${ }^{11,12,14}$ Another example is that among the cubic, cuboctahedral, and octahedral nanocrystals of Ag, the octahedral nanocrystals display the highest Raman scattering enhancement factor and the best sensitivity for trace amounts of arsenic in contaminated water. ${ }^{17}$ Therefore, shape control of gold nanoparticles is a critical area of research that can lead to the rational design of their catalytic and optical properties. $^{18}$

According to the Winterbottom construction, ${ }^{19}$ also known as the Wulff-Kaishew theorem, ${ }^{20}$ the substrate plays an important role in determining the aspect ratio of a crystal via the interfacial energy $\left(\gamma_{\mathrm{i}}\right)$ and the substrate surface energy $\left(\gamma_{\mathrm{s}}\right)$. The energy $\gamma^{*}$ is defined as $\gamma^{*}=\gamma_{\mathrm{i}}-\gamma_{\mathrm{s}},{ }^{21}$ i.e., it is the net energy 
change at the interface when the crystal is attached to the substrate. Higher values of $\gamma^{*}$ result in taller crystals (i.e., a higher height-to-width ratio). ${ }^{22,23}$ In many studies the experimental aspect ratio of nanocrystals were used to calculate $\gamma^{*}$ and the energies associated with it, e.g., $\gamma_{\mathrm{i}}, \gamma_{\mathrm{s}}$, and the adhesion energy $\gamma_{\text {adh. }}{ }^{21,24-28}$ However, it has been demonstrated by some theoretical studies that the crystal aspect ratio is not a constant with volume if substrate-induced epitaxial strain is present. ${ }^{29-32}$

Gold nanoparticles used in catalysis are usually prepared on oxide substrates, which can serve as a template for nanocrystal epitaxy. ${ }^{33}$ More importantly, the atomic structure, chemistry, and surface energy of the substrate surface are all determining factors of the nanocrystal morphology. ${ }^{21-24,34-36}$ The substrates used in our work are strontium titanate $\left(\mathrm{SrTiO}_{3}\right)$ single crystals epi-polished on the (001) and (111) surfaces. $\mathrm{SrTiO}_{3}$ is able to accommodate a large variety of surface reconstructions with different compositions and surface chemistry. In our study, three surfaces were prepared: $\mathrm{SrTiO}_{3}(001)-(2 \times 1),{ }^{37,38}$ $\mathrm{SrTiO}_{3}(001)-c(4 \times 2),{ }^{37,39,40}$ and $\mathrm{SrTiO}_{3}(111)-(4 \times 4)+(6 \times 6) .{ }^{41,42}$ These surfaces are found to modify the gold crystal shapes through interfacial strain, by promoting the growth of $\{111\}$ and $\{001\}$ crystal facets differently, and by encouraging reentrant facets to grow out. These findings are a key step towards the controlled synthesis of nanocrystal shapes, which is important for optimizing their properties for the development of catalytic materials, biological sensors, and photonic devices. ${ }^{43}$

\section{Experimental Methods}

The substrates for gold nanocluster growth are $\mathrm{SrTiO}_{3}$ single crystals doped with $\mathrm{Nb}$ at $0.5 \%$ by weight, supplied by PI-KEM, U.K. $\mathrm{SrTiO}_{3}(001)-(2 \times 1)$ was prepared by annealing in UHV at $900-950{ }^{\circ} \mathrm{C}$ for $1 \mathrm{~h}^{37} \mathrm{SrTiO}_{3}(001)-c(4 \times 2)$ was produced by $\mathrm{Ar}^{+}$-ion sputtering at $500 \mathrm{eV}$ for $10 \mathrm{~min}$, followed by 
annealing at $1100-1150{ }^{\circ} \mathrm{C}$ for $30 \mathrm{~min}$ in $\mathrm{UHV}^{37,40} \mathrm{SrTiO}_{3}(111)-(4 \times 4)+(6 \times 6)$ was generated by $\mathrm{Ar}^{+}-$ ion sputtering at $500 \mathrm{eV}$ for $6 \mathrm{~min}$ and subsequent $\mathrm{UHV}$ annealing at $1090{ }^{\circ} \mathrm{C}$ for $1.5 \mathrm{~h} .{ }^{41}$ Gold was deposited onto $\mathrm{SrTiO}_{3}$ substrates (held at $300-400{ }^{\circ} \mathrm{C}$, unless otherwise stated) in UHV using a Createc Knudsen cell heated at $1350{ }^{\circ} \mathrm{C}$. The deposition typically occurred at a rate of $\sim 0.025$ monolayers $\min ^{-}$ 1.

The reconstructed $\mathrm{SrTiO}_{3}$ substrates and gold islands were imaged by scanning tunneling microscopy (STM) (JEOL JSTM 4500s model, base pressure $10^{-8} \mathrm{~Pa}$ ). STM images were processed by Smart Align, ${ }^{44,45}$ Gwyddion, and $W S x M{ }^{46}$ The gold crystals were also imaged by a Zeiss Merlin scanning electron microscope (SEM) at accelerating voltages of $2-3 \mathrm{kV}$.

\section{Results and discussion}

\subsection{Crystal epitaxy}

Fig. 1 shows STM images of gold islands on the three different $\mathrm{SrTiO}_{3}$ substrates: $(\mathrm{a}, \mathrm{b}) \mathrm{SrTiO}_{3}(001)-(2$ $\times 1),(\mathrm{c}, \mathrm{f}) \mathrm{SrTiO}_{3}(001)-c(4 \times 2)$, and $(\mathrm{d}, \mathrm{e}) \mathrm{SrTiO}_{3}(111)-(4 \times 4)+(6 \times 6)$. On all three substrates, the gold nanocrystals have a (111) base and a (111) top facet of the truncated triangular shape. On $\mathrm{SrTiO}_{3}(001)$ $(2 \times 1)$, the crystal heights are labeled below each crystal (Fig. 1a,b), where it can be seen that in addition to the gold nanocrystals, a few irregularly shaped, flat gold islands are also present. A few examples are indicated by gray arrows. These islands are gold monolayers, which have previously been reported on $\mathrm{SrTiO}_{3}(001)-(2 \times 1) .{ }^{28}$ We confirm that they are not seen on any other surface of $\mathrm{SrTiO}_{3}$, and will not be discussed further here. On $\mathrm{SrTiO}_{3}(001)-c(4 \times 2)$ and $\mathrm{SrTiO}_{3}(111)-(4 \times 4)+(6 \times 6)$, multiply twinned particles (MTPs) are observed in addition to nanocrystals, the coexistence of which is shown in Fig. 1e, 
where all nanocrystals are indicated by white arrows. The MTPs are supported icosahedra, which are polyhedra with 20 faces in their free-standing form (sketched in Fig. 1f). The MTPs in Fig. 1e adopt various orientations of icosahedra, and the three main ones are shown in the close-up images in Fig. 1f, termed as point $(\mathrm{P})$, face $(\mathrm{F})$, and edge $(\mathrm{E})$ orientations in previous reports. ${ }^{28,47}$ These MTPs only have $\{111\}$ facets, the lowest-energy termination. Therefore they are a particle form with a reduced surface energy at the cost of strain and twinning energies, usually observed at smaller sizes than their singlecrystal counterparts. ${ }^{48}$ MTPs are the nucleation shape of gold islands on substrate surfaces other than $\mathrm{SrTiO}_{3}(001)-(2 \times 1)$, where the substrate does not satisfy the condition to stabilize monolayers.
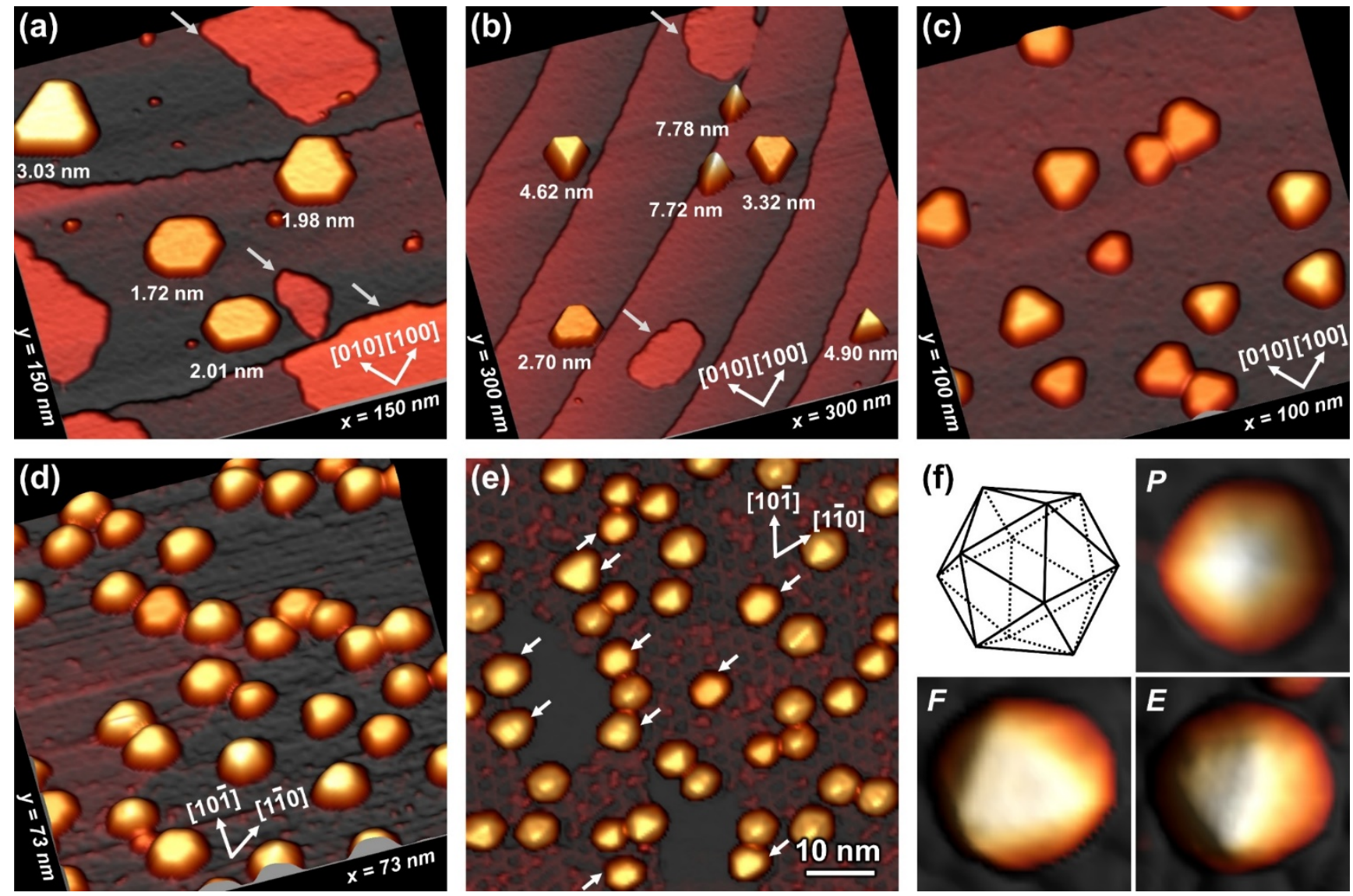

Fig. 1: 3D STM images of gold islands on $(\mathrm{a}, \mathrm{b}) \mathrm{SrTiO}_{3}(001)-(2 \times 1)\left(\mathrm{a}: V_{\mathrm{s}}=1.0 \mathrm{~V}, I_{\mathrm{t}}=0.05 \mathrm{nA}\right.$; b: $V_{\mathrm{s}}=1.5 \mathrm{~V}$, $\left.I_{\mathrm{t}}=0.10 \mathrm{nA}\right),(\mathrm{c}, \mathrm{f}) \mathrm{SrTiO}_{3}(001)-c(4 \times 2)\left(\right.$ both $\left.V_{\mathrm{s}}=4.0 \mathrm{~V}, I_{\mathrm{t}}=0.05 \mathrm{nA}\right)$, and $(\mathrm{d}, \mathrm{e}) \mathrm{SrTiO}_{3}(111)-(4 \times 4)+(6 \times 6)$ $\left(\mathrm{d}: V_{\mathrm{s}}=3.8 \mathrm{~V}, I_{\mathrm{t}}=0.03 \mathrm{nA}\right.$; e: $\left.V_{\mathrm{s}}=4.0 \mathrm{~V}, I_{\mathrm{t}}=0.10 \mathrm{nA}\right)$. In $(\mathrm{a}, \mathrm{b})$, gold monolayers are indicated by gray arrows and the crystal heights are labeled below each crystal. In (e), gold MTPs coexist with crystals. All crystals are indicated by white arrows. Three close-up images of MTPs are shown in (f), with point (P), face (F), and edge (E) orientations. A free-standing MTP is also sketched. 
For the purpose of epitaxy and strain analysis, it is helpful to clarify the crystallography of gold on $\mathrm{SrTiO}_{3}$. Fig. 2a shows a cubic unit cell of gold with a face-centered-cubic (fcc) structure, with a lattice constant of $4.078 \AA$. The closest $\mathrm{Au}-\mathrm{Au}$ periodicity is therefore $4.078 / \sqrt{ } 2=2.884 \AA$. In the STM images of gold crystals in Fig. 1, their truncated triangular top facets indicate that gold forms the crystal shape modeled in Fig. 2b,c, which has (111) top and base facets and $\{111\}$ and $\{001\}$ side facets. It can be seen from Fig. $2 c$ that the six edges of the crystal all align with the $<1 \overline{1} 0>$-type directions of gold. A single $\mathrm{Au}(111)$ layer is 6-fold symmetric, while a 3D fcc packed structure reduces it to 3-fold. Therefore, the three longer and the three shorter crystal edges in Fig. 2c are not equivalent to one another. Bulk $\mathrm{SrTiO}_{3}$ possesses a cubic lattice above $105 \mathrm{~K}(a=3.905 \AA),{ }^{49}$ and its unit cell is shown in Fig. $2 \mathrm{~d}$. The $\mathrm{Sr}^{2+}$ ion sits in a site that is 12 -fold-coordinated by $\mathrm{O}^{2-}$ ions, and the $\mathrm{Ti}^{4+}$ ions are octahedrally coordinated with respect to the $\mathrm{O}^{2-}$ ions.

From STM images like Fig. 1a-c, the gold crystal orientations on $\mathrm{SrTiO}_{3}(001)$ are measured according to the diagram at the top of Fig. 2e. The following histograms present the results on $\mathrm{SrTiO}_{3}(001)-(2 \times$ 1) (upper) and $-c(4 \times 2)$ (lower). In both, two peaks are identified at $0^{\circ}$ and $30^{\circ}$, relative to the substrate [100] direction. They are two crystallographically equivalent orientations because of the 4-fold symmetry of $\mathrm{SrTiO}_{3}(001)$. Therefore, the preferred epitaxial orientation of gold crystals on $\mathrm{SrTiO}_{3}(001)$ is such that their three longer $<1 \overline{1} 0>$ edges align with the $<100>$ directions on $\mathrm{SrTiO}_{3}(001)$. This results in four epitaxially equivalent footprints of gold crystals on $\mathrm{SrTiO}_{3}(001)$ shown in Fig. 2f, two of which correspond to the $0^{\circ}$ peak and the other to the $30^{\circ}$ peak. The interfacial crystallographic relationship can

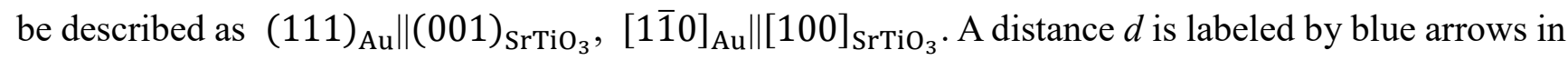
Fig. 2f, which describes the coincidence epitaxy: four unit cells (bond lengths) of gold match three unit cells along $\mathrm{SrTiO}_{3}[100]: d=4 \times 2.884 \AA=11.536 \AA \approx 3 \times 3.905 \AA=11.715 \AA$, with a $1.5 \%$ tensile misfit strain in gold. 


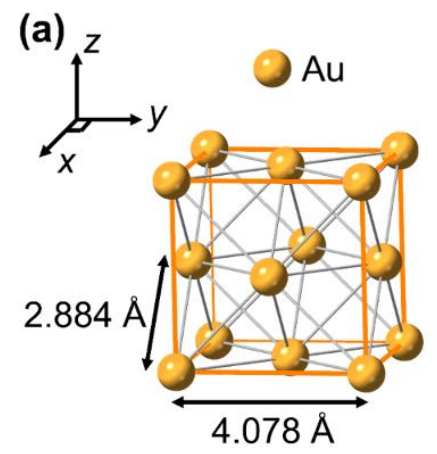

(e)
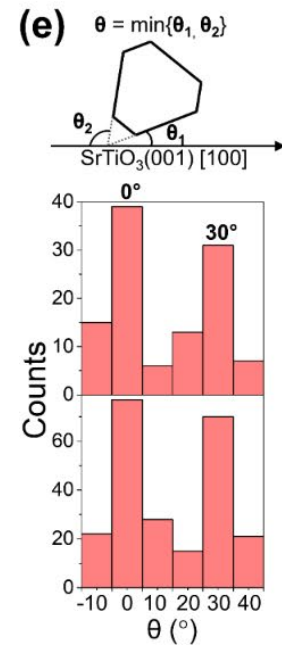

(b)

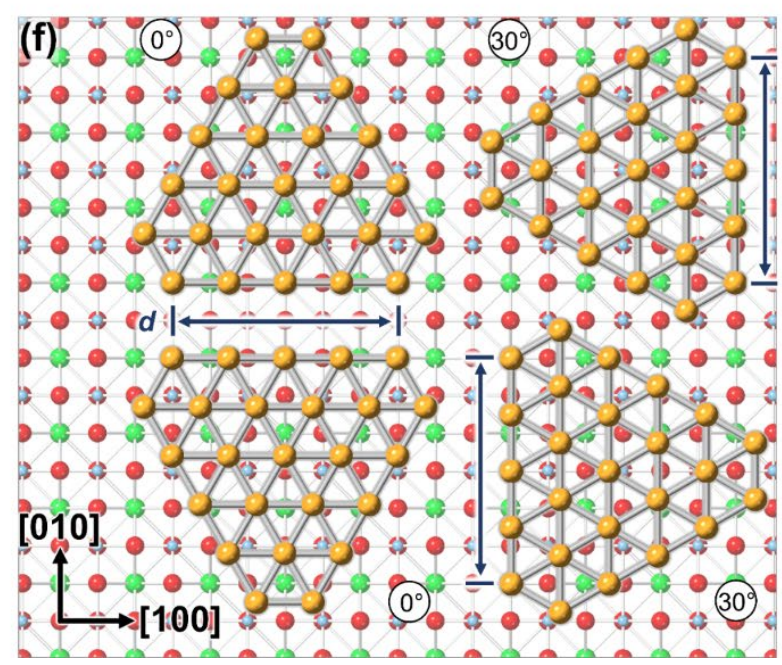

(c)

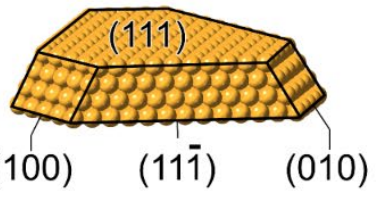

(010)

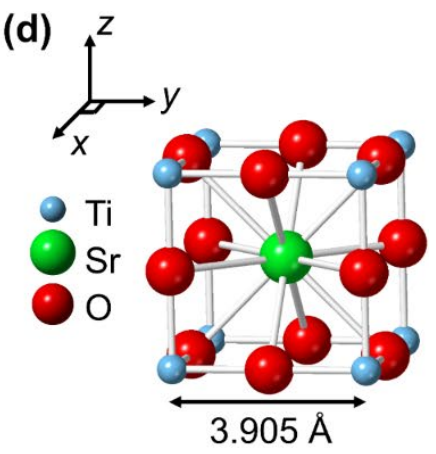

(g)
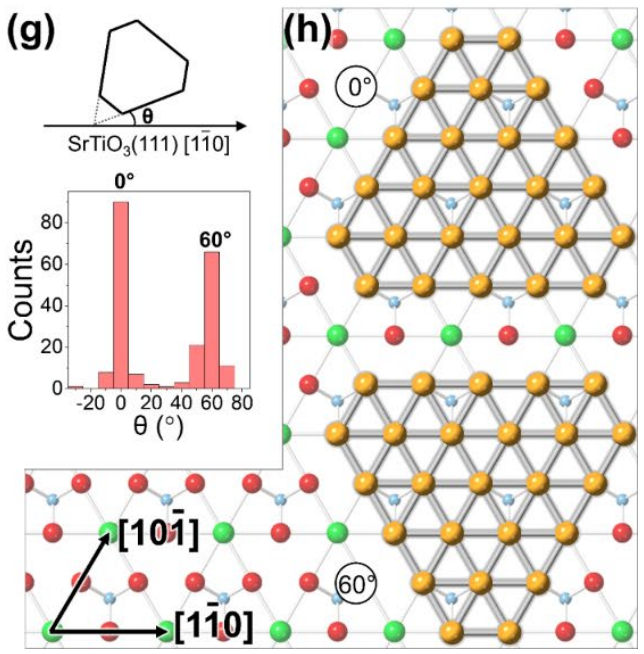

Fig. 2: Crystal structures of gold on $\mathrm{SrTiO}_{3}$. (a) Fec unit cell of gold outlined in orange, with a lattice constant of $4.078 \AA$. The $A u-A u$ bonds are in gray with a bond length of $2.884 \AA$. (b,c) Space-filling atomic models of a gold crystal with a (111) top facet (tilted and top views). In the top view, the six crystal edges align with the $<\mathbf{1} \overline{1} 0>$-type directions of gold. (d) Unit cell of $\mathrm{SrTiO}_{3}$. (e) Orientation histograms of gold crystals on $\mathrm{SrTiO}_{3}(001)-(2 \times 1)$ (upper) and $-c(4 \times 2)$ (lower), measured according to the diagram above. (f) Four preferred orientations of gold crystals on $\mathrm{SrTiO}_{3}(001)$. $d$ = four Au unit lengths (bond lengths) = three $\mathrm{SrTiO}_{3}[100]$ unit lengths. The substrate lattice directions are labeled. For clarity, only one layer of gold atoms is shown. (g) Orientation histogram of gold crystals on $\mathrm{SrTiO}_{3}(111)-(4 \times 4)+(6 \times 6)$, measured according to the diagram above. (h) Two preferred orientations of gold crystals on $\mathrm{SrTiO}_{3}(111)$. The substrate lattice directions are labeled. For clarity, only one layer of gold atoms and two layers of atoms in $\mathrm{SrTiO}_{3}(111)$ are shown.

On $\mathrm{SrTiO}_{3}(111)-(4 \times 4)+(6 \times 6)$, the gold crystals tend to align their longer $<1 \overline{1} 0>$ edges with the threefold symmetric $<1 \overline{1} 0>$ directions on $\mathrm{SrTiO}_{3}$ (Fig. 1d). This is statistically illustrated by the histogram in Fig. 2 g. The two peaks at $0^{\circ}$ and $60^{\circ}$ correspond to the two crystal orientations drawn in Fig. $2 \mathrm{~h}$. The coincidence epitaxy results from the similar lattice constants of gold and $\mathrm{SrTiO}_{3}: 4.078 \AA \approx 3.905 \AA$, with a $4.4 \%$ compressive misfit strain in gold. In addition to the lattice commensuration, $\mathrm{Au}(111)$ and $\mathrm{SrTiO}_{3}(111)$ are also symmetry coincident. This means that the epitaxial match between them is more 
powerful (two-dimensional) because there is epitaxy in both the [10 $\overline{1}]$ and the [1 $\overline{1} 0]$ directions on $\mathrm{SrTiO}_{3}(111)$. Conversely, the match between $\mathrm{Au}(111)$ and $\mathrm{SrTiO}_{3}(001)$ is one-dimensional because there is only epitaxy along one of the $<100>$ directions on $\mathrm{SrTiO}_{3}(001)$. The stronger epitaxial match can be visually seen from the better atomic overlap in Fig. $2 \mathrm{~h}$ than in Fig. $2 \mathrm{f}$.

The epitaxial relationship on $\mathrm{SrTiO}_{3}(111)$ can be described as $(111)_{\mathrm{Au}} \|(111)_{\mathrm{SrTiO}_{3}}$, $[1 \overline{1} 0]_{\mathrm{Au}} \|[1 \overline{1} 0]_{\mathrm{SrTiO}_{3}}$. Note that in Fig. $2 \mathrm{~h}$, although only one layer of gold atoms is included for clarity, multilayer gold crystals are 3-fold symmetric as explained earlier. Since the $\mathrm{SrTiO}_{3}(111)$ substrate is 3fold symmetric as well, the orientations of the two gold crystals in Fig. $2 \mathrm{~h}$, differing by $60^{\circ}$, are not equivalent. Their inequivalence can also be seen from an SEM image of gold crystals on $\mathrm{SrTiO}_{3}(111)$ $(4 \times 4)+(6 \times 6)($ later in Fig. $5 f)$, in which the crystals have only one exclusive orientation. The large crystals were post-annealed at $600{ }^{\circ} \mathrm{C}$ for $5 \mathrm{~h}$, and they are more likely to have reached a thermodynamically optimized state than the smaller crystals observed in STM without post-deposition thermal treatments.

When the gold crystals are high enough, their Wulff shape suggests that they should form reentrant facets (Fig. 3a). However, in our study these reentrant facets grow out and the $\{001\}$ facets extend all the way down to the substrate, as shown in Fig. 3b. The reason for the formation of this "growth shape" will be explained later (section 3.4). Prior to presenting our experimental results, it is useful to define three dimensions that characterize the crystal growth shape (Fig. 3b): $l$ is the width across the top (111) facet measured from the middle of one $\{001\}$ side facet to the middle of the opposite $\{111\}$ side facet; $s$ is the width of a $\{001\}$ facet; $h$ is the crystal height. From the STM images in Fig. 1 , it can be seen that the gold crystals on various substrates differ slightly in their shapes, e.g., some are more pointed (smaller $s$ ) and some are closer to hexagons (larger $s$ ). 
(a)
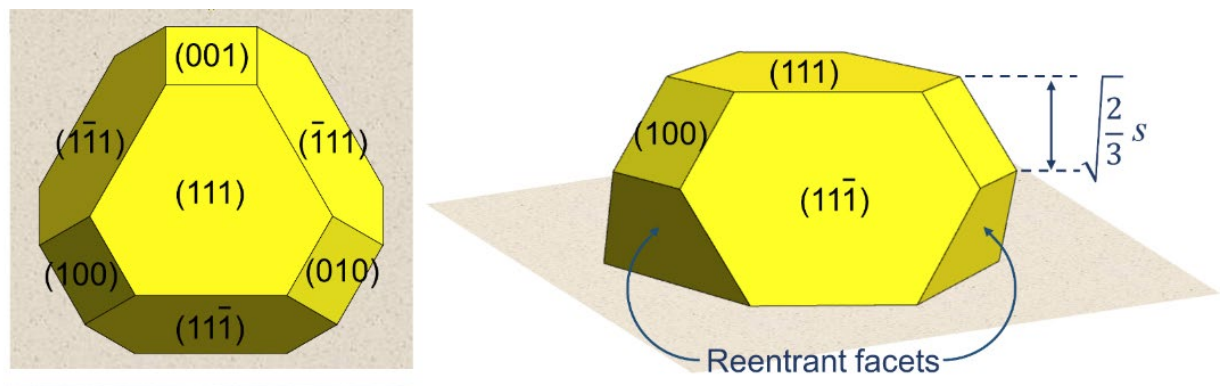

(b)
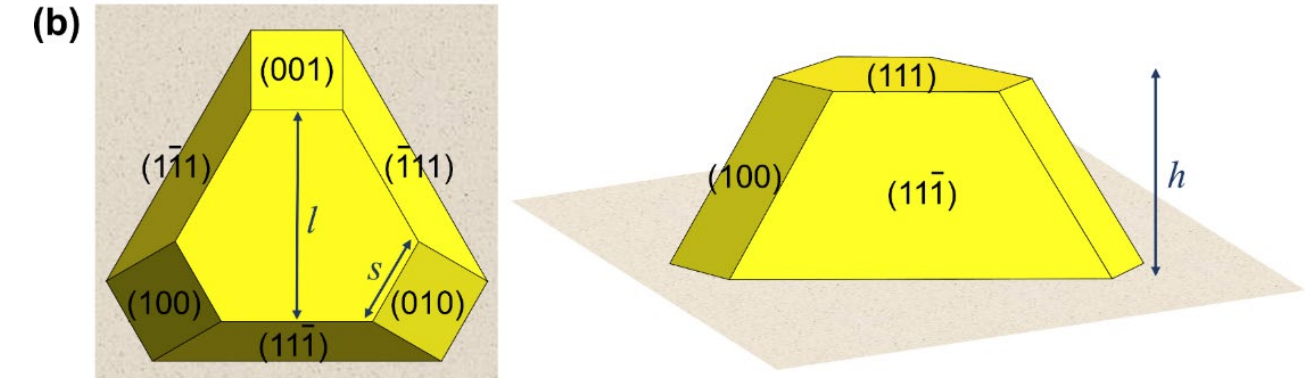

Fig. 3: (a) Wulff and (b) growth shapes of gold crystals on a substrate: top view (left) and tilted view (right). The three important crystal dimensions, $l, s$, and $h$ are labeled in (b). In (a), once the crystal height exceeds $\sqrt{2 / 3} s$, the Wulff shape has reentrant facets.

\subsection{Height-to-width ratio $(h / l)$}

For a crystal supported on a lattice-mismatched substrate, its total energy has three contributions: the formation energy (negative), the surface/interface energies (positive), and the strain energy due to the lattice mismatch (positive). ${ }^{29,30,50}$ In the absence of any interfacial misfit strain, e.g., on a lattice-matched or amorphous substrate, the thermodynamically optimized shape of a gold crystal is predicted by minimizing its surface/interface energies. This is described by the Winterbottom construction, in which a free crystal with the Wulff shape is truncated by the substrate. ${ }^{19,20}$ The height-to-width ratio $(h / l)$ should then be a constant on a given substrate.

The $h / l$ ratio of gold crystals on the three $\mathrm{SrTiO}_{3}$ substrates is calculated from measurements and plotted against the crystal height $h$ (Fig. 4a). The gold crystals chosen to be measured were all grown on a 
substrate held at $300{ }^{\circ} \mathrm{C}$ or above to best ensure that the crystals were kinetically capable of reaching their equilibrium shapes. Nevertheless, the data in Fig. 4a represent crystals that are both growing and shrinking due to the Ostwald ripening phenomenon. We find that on all three substrates there is a positive relationship between $h / l$ and $h$ and a red linear regression line is fitted in each case to guide the eye. On $\mathrm{SrTiO}_{3}(111)-(4 \times 4)+(6 \times 6)$, when $h>4 \mathrm{~nm}$ the $h / l$ ratio starts to level out and these data points were left out when the linear trendline was fitted. The fact that a higher crystal has a higher $h / l$ ratio cannot be explained by kinetic arguments, because kinetics would favor the opposite: the higher a crystal, the more difficult for additional atoms to climb up the side facets, which in turn means a lower $h / l$ ratio.

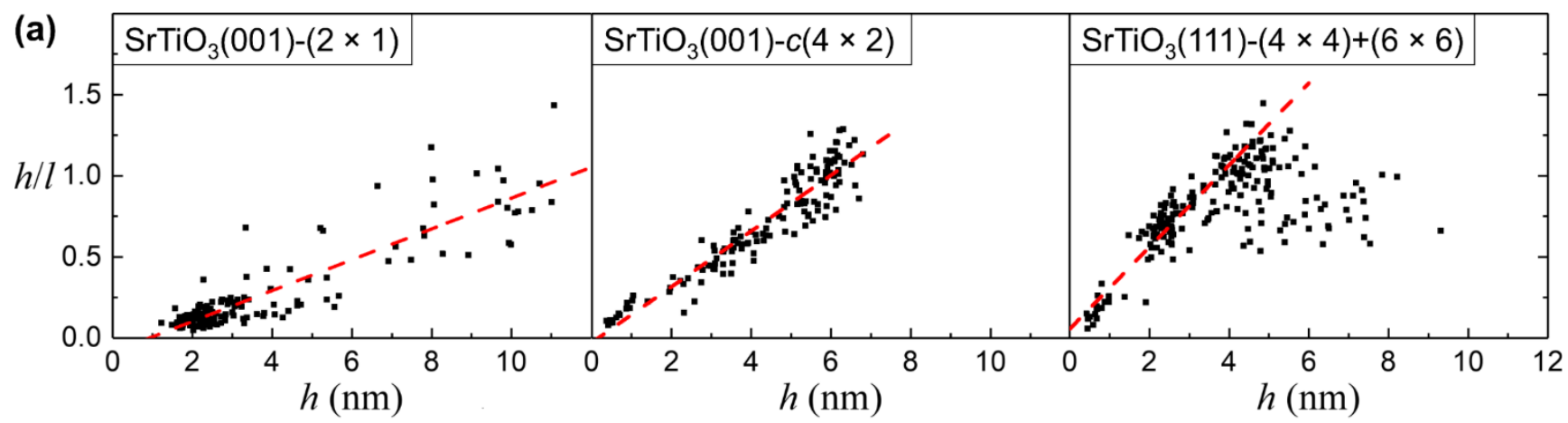

(b)

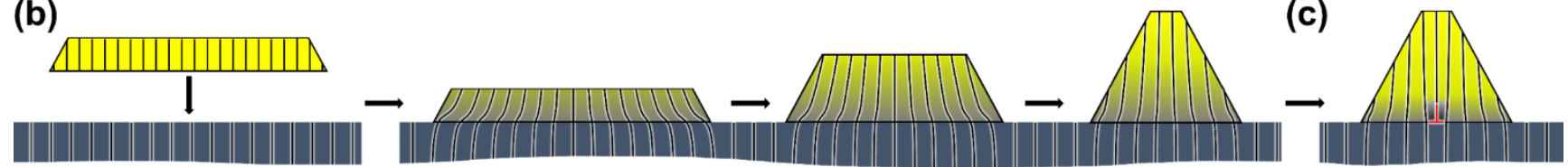

Fig. 4: Height-to-width $(h / l)$ ratio of gold crystals and schematic sketches. (a) $h / l$ plotted against crystal height on $\mathrm{SrTiO}_{3}(001)-(2 \times 1), \mathrm{SrTiO}_{3}(001)-c(4 \times 2)$, and $\mathrm{SrTiO}_{3}(111)-(4 \times 4)+(6 \times 6)$. A linear regression line (red) is fitted to each dataset. (b,c) Schematic drawings of gold crystals (yellow) and the substrate (dark blue). The vertical black lines represent lattice planes. (b) Heightening of the gold crystal, which helps to relieve the strain resulting from the interfacial lattice misfit. The strain field strength is indicated by the shade of gray. (c) A strained crystal reducing its strain by losing full coherency and forming a misfit dislocation (red) at the interface.

The non-constant $h / l$ ratio is explained by the lattice misfit at the $\mathrm{Au}-\mathrm{SrTiO}_{3}$ interface, which is $1.5 \%$ tensile in gold on $\mathrm{SrTiO}_{3}(001)$ and 4.4\% compressive in gold on $\mathrm{SrTiO}_{3}(111)$. Since our gold crystals were grown at substrate temperatures of $300-400{ }^{\circ} \mathrm{C}$ (and occasionally post-annealed at $500-600{ }^{\circ} \mathrm{C}-$ 
see Fig. 5b-f), an additional interfacial strain develops due to the different thermal expansion coefficients of gold $\left(1.42 \times 10^{-5} \mathrm{~K}^{-1}\right)^{51}$ and $\mathrm{SrTiO}_{3}\left(3.23 \times 10^{-5} \mathrm{~K}^{-1}\right) .{ }^{52}$ However, given our treatment temperatures, the magnitude of this thermally induced strain is up to $\sim 1 \%$ only. Misfit strains of such magnitude (a few percent in total) have been observed to be accommodated with full coherency between $\mathrm{Au}$ and $\mathrm{TiO}_{2},{ }^{35}$ and this is also likely to be the case in our Au-on-SrTiO 3 system, certainly for small crystals.

The $h / l$ ratio of gold crystals increases with the crystal height (Fig. 4a) because crystal heightening can help to release the interfacial strain, ${ }^{29-32}$ as shown by the schematic drawings in Fig. 4b. The gold crystals (yellow) are strained by the lattice-mismatched substrate (dark blue), where the interfacial atoms are displaced from their ideal lattice positions. The strain field (gray) decays as it moves away from the interface. The black lines represent the lattice planes, which become vertical if the epitaxial strain is fully relieved. The average strain field within the gold crystal is reduced by crystal heightening, e.g., the top facet of the highest crystal in Fig. $4 \mathrm{~b}$ is close to being strain-free.

The gradients of the fitted trendlines in Fig. $4 \mathrm{a}$ indicate the rates at which the $h / l$ ratios increase with $h$. This rate is the smallest on $\mathrm{SrTiO}_{3}(001)-(2 \times 1)(0.095 \pm 0.003)$, followed by $\mathrm{SrTiO}_{3}(001)-c(4 \times 2)$ $(0.172 \pm 0.005)$, and the greatest on $\mathrm{SrTiO}_{3}(111)-(4 \times 4)+(6 \times 6)(0.253 \pm 0.011)$. There is a correlation between these rates and four factors: (1) the $\mathrm{Au}-\mathrm{SrTiO}_{3}$ interfacial energy, (2) the degree of epitaxial lattice mismatch, (3) whether the epitaxial match is along only one or both crystal directions on the substrate surface, and (4) the relative stiffness of the substrate compared to that of the crystal. ${ }^{31,32}$ The fourth factor can be assumed to very similar for all three substrates as the bulk material is the same.

The $\mathrm{SrTiO}_{3}(111)-(4 \times 4)+(6 \times 6)$ substrate displays the highest gradient of $h / l$ for two reasons. Firstly, it has a higher misfit strain with gold (4.4\%) than the other two substrates (1.5\%). Secondly, the epitaxial 
match on $\mathrm{SrTiO}_{3}(111)$ is more powerful (2D) than that on $\mathrm{SrTiO}_{3}(001)$ (1D) (Fig. 2f,h). The gradients on $\mathrm{SrTiO}_{3}(001)-(2 \times 1)$ and $-c(4 \times 2)$ also differ because the surface energy $\left(\gamma_{\mathrm{s}}\right)$ of $(2 \times 1)$ is higher than that of $c(4 \times 2)$ by $0.4-0.6 \mathrm{~J} \mathrm{~m}^{-2} \cdot{ }^{24,53}$ The higher $\gamma_{\mathrm{s}}$ encourages a higher degree of wetting, i.e., a lower value of $h / l$ at a given height $h$, and hence a lower gradient of $h / l$.

In Fig. 4a, the "anomalous" points when $h>4 \mathrm{~nm}$ on $\mathrm{SrTiO}_{3}(111)-(4 \times 4)+(6 \times 6)$ can be explained by interfacial dislocations: at a critical thickness of $\sim 4 \mathrm{~nm}$, the accumulated strain energy eventually induces plastic relaxation at the $\mathrm{Au}-\mathrm{SrTiO}_{3}$ interface. ${ }^{29,31,32}$ This is illustrated by the sketch in Fig. 4c, in which the coherent (dislocation-free) island partially relieves the misfit strain by introducing a misfit dislocation at the interface, drawn in red. The dislocation is introduced at the cost of additional strain energy around the dislocation core, which is shown schematically by the darker area around the dislocation in Fig. 4c. On $\mathrm{SrTiO}_{3}(001)-(2 \times 1)$ and $-c(4 \times 2)$, the linear trend of $h / l$ remains up to the highest gold crystals we observed, suggesting that the critical thickness for dislocation introduction has not been reached on $\mathrm{SrTiO}_{3}(001)$ at up to $h=12 \mathrm{~nm}$. The earlier introduction of dislocations on the $\mathrm{SrTiO}_{3}(111)-(4 \times 4)+(6 \times 6)$ substrate is due to two factors mentioned earlier: (i) the greater misfit strain (4.4\% versus $1.5 \%)$ and (ii) the stronger epitaxy (2D versus $1 \mathrm{D})$.

For comparison, in our previous study, ${ }^{28}$ the $h / l$ ratio of gold crystals on $\operatorname{SrTiO}_{3}(001)-(2 \times 1)$ was reported to be a constant with crystal volume: $0.42 \pm 0.05$. Measurements were made of 22 crystals in total and the largest one was just over $1000 \mathrm{~nm}^{3}$ in volume. Here, in Fig. 4a we present 154 data points on $\mathrm{SrTiO}_{3}(001)-(2 \times 1)$ and the crystals span a greater size range: about half of them are over $1000 \mathrm{~nm}^{3}$ in volume and the largest one is $4030 \pm 94 \mathrm{~nm}^{3}$. The gold crystals measured in the previous paper correspond to a smaller range of data $(h \sim 1.7-3.4 \mathrm{~nm})$ with $h / l=0.42 \pm 0.05,{ }^{28}$ and the constant $h / l$ ratio reported may simply due to the limited range. Nevertheless, within the same height range (1.7-3.4 nm), 
our average value of $h / l$ is $0.14 \pm 0.08$, which is noticeably smaller than 0.42 . This difference can be attributed to our different growth parameters: in the previous paper, 0.8 monolayer of gold was deposited onto the substrate heated to $400{ }^{\circ} \mathrm{C},{ }^{28}$ in this paper, $0.25-0.50$ monolayer of gold ( $66 \%$ at 0.25 monolayer) was deposited onto the substrate kept at $300-400{ }^{\circ} \mathrm{C}$. The main difference is the larger deposition amount of gold in the previous study, which resulted in a visibly higher density of the gold crystals. ${ }^{28} \mathrm{~A}$ higher density of crystals means that a greater proportion of the evaporated gold lands directly on the top facets of the growing crystals, hence resulting in a greater $h / l$ ratio. The difference in the $h / l$ ratios of the two datasets serves to illustrate how sensitive this ratio is to the parameters under which the experiments are performed. This point is discussed in detail in the review by Marks and Peng, ${ }^{43}$ who point out that each nanocrystal in a distribution is either growing or shrinking, and may even be changing form, e.g., from an fcc crystal to an MTP. This is a result of Ostwald ripening, during which larger particles grow at the expense of smaller ones. This phenomenon occurs continuously, though only at a noticeable rate at elevated temperatures. Therefore, the gold crystals observed and measured only represent a snapshot of their evolution towards absolute thermodynamic equilibrium. This means that thermodynamic models are only ever an approximation to experimentally derived data.

Accordingly, the gold crystals should ideally be treated at high temperatures (e.g., close to their melting point) to promote the mobility of gold atoms, to allow the gold crystals to evolve towards their thermodynamically equilibrium shape as much as possible. This is the reason why we held the substrates at above $300{ }^{\circ} \mathrm{C}$ during gold crystal growth. However, the substrate temperature was usually kept below $400{ }^{\circ} \mathrm{C}$ because higher growth temperatures resulted in crystals that were too high to be scanned by the STM tip, e.g., when $h>\sim 10 \mathrm{~nm}$. Nevertheless, we annealed a few samples at $500-600{ }^{\circ} \mathrm{C}$ for SEM studies (see Fig. 5b-f), which indeed enhanced the kinetics towards equilibrium. For example, as mentioned in section 3.1, the gold crystals on $\mathrm{SrTiO}_{3}(111)-(4 \times 4)+(6 \times 6)$ grown at $300-400{ }^{\circ} \mathrm{C}$ mainly 
adopt two orientations (Fig. 1d and Fig. 2g,h), which are known to be inequivalent because of the 3fold symmetry of both gold and $\mathrm{SrTiO}_{3}(111)$. After the gold crystals were post-annealed at $600{ }^{\circ} \mathrm{C}$, only one exclusive orientation was observed (Fig. 5f), which must be the thermodynamically favored epitaxial orientation.

It is widely reported in the literature that for a crystal grown on a lattice-mismatched substrate, the interfacial strain opposes wetting and leads to a higher $h / l$ ratio, though most of the past studies are computational. ${ }^{29-32}$ Therefore, when we minimize the total energy of a gold crystal to thermodynamically optimize its shape, we need to include its strain energy in addition to its surface/interface energies. The strain energy is a complex function of the crystal and substrate stiffness, the lattice misfit between them, the crystal volume, and the crystal shape. ${ }^{29,32} \mathrm{~A}$ few reports also mention that the $h / l$ ratio increases with increasing lattice misfit, increasing strain-free $h / l$ ratio, and increasing substrate/crystal stiffness ratio. ${ }^{31,32}$ These are consistent with our observations for the three $h / l$ ratio gradients on the three $\mathrm{SrTiO}_{3}$ substrates.

Additionally, the strain should propagate from the interface into both the crystal and the underlying substrate, ${ }^{29,31,32}$ but for simplicity Fig. 4b,c only shows the strain field (gray) in the gold crystals. Gold has a relatively low Young's modulus of $E(\mathrm{Au})=79 \mathrm{GPa},{ }^{54}$ compared with $E_{<100>}\left(\mathrm{SrTiO}_{3}\right)=225-$ $265 \mathrm{GPa}^{55,56}$ Hence we expect that the interfacial misfit strain would be largely taken up in the gold crystals. This is also due to the small size of gold crystals that allows them to relax more easily parallel to the substrate surface plane.

It has also been reported that as the interfacial strain energy accumulates in an epitaxially substratesupported crystal, interfacial dislocations will be introduced at a critical thickness. ${ }^{29,31,32}$ In our Au-on$\mathrm{SrTiO}_{3}$ system, dislocations are more likely to be generated in gold than in $\mathrm{SrTiO}_{3}$ (as drawn in Fig. 4c). 
This is because the dislocation energy per unit length is $\propto G b^{2}$, where $G$ is the shear modulus and $\boldsymbol{b}$ is the Burgers vector. These values are both smaller in gold: $G(\mathrm{Au})=27 \mathrm{GPa},{ }^{54} G_{<100>}\left(\mathrm{SrTiO}_{3}\right)=$ $121 \mathrm{GPa},{ }^{56} b(\mathrm{Au})=2.884 \AA$, and $b\left(\mathrm{SrTiO}_{3}\right)=3.905 \AA$.

When a dislocation is introduced, it partially releases the interfacial strain and abruptly modifies the equilibrium shape, resulting in a reduced $h / l$ ratio. Thermodynamically the growing crystal will have an oscillating or sawtooth $h / l$ ratio as successive dislocations are introduced. ${ }^{31,32,57}$ This behavior has been calculated for the Ge-on-Si(001) system. ${ }^{57}$ In our Au-on- $\mathrm{SrTiO}_{3}(111)$ system, the period of oscillation in the gold crystal basal plane width is calculated to be $5.49 \mathrm{~nm}$ (= the strain-free dislocation separation), which corresponds to an oscillation period in the crystal height of $5.18 \mathrm{~nm}$. In Fig. 4a, on $\mathrm{SrTiO}_{3}(111)$ $(4 \times 4)+(6 \times 6)$ the nucleation of the first dislocations occurs at $h \approx 4 \mathrm{~nm}$, upon which the $h / l$ ratio drops. Thereafter, the $h / l$ ratio should rise until $h \approx 4+5.18 \approx 9 \mathrm{~nm}$, when it should suddenly drop again. Our data are not of a sufficient quality to clearly show this oscillation. However, there are three misfit dislocations introduced per $5.18 \mathrm{~nm}$ period, along the three $<1 \overline{1} 0>$ directions in the gold (111) plane. Unless they are all introduced at the same time, the dramatic drop in the $h / l$ ratio will not be easily observed. On the $\mathrm{SrTiO}_{3}(001)$ substrates, the oscillation period in the crystal base width is calculated to be $18.74 \mathrm{~nm}$, corresponding to a period of $17.67 \mathrm{~nm}$ in the crystal height. These values fall outside our data range in Fig. 4a.

Finally, we comment that although the $h / l$ ratio is not a constant due to the interfacial strain, in some studies, e.g., of Pt and Pd nanocrystals, ${ }^{58,59}$ the "interfacial energy" was still calculated from the nanocrystal geometry based on the Winterbottom construction. As the crystal aspect ratio was also observed to heighten, the "interfacial energy" appears to increase with increasing crystal height, which was explained by the interfacial strain and strain relief by dislocations. ${ }^{58}$ However, we do not think that 
in our case this is a helpful analysis method because the interfacial energy is an area-dependent term that increases in magnitude as misfit dislocations are introduced, whereas the strain is a volumedependent term that decreases when strain-relieving misfit dislocations are introduced.

\section{3 $A_{111} / A_{001}$ or $s / l$ ratio}

In addition to the $h / l$ ratio, the other geometric ratio that together defines the gold crystal shape is the ratio between $s$ and $l$ (Fig. 3b). The $s / l$ ratio is a direct measure of the ratio between the $\{111\}$ and $\{001\}$ surface areas $\left(A_{111} / A_{001}\right)$. This depends on the surface energies of gold, $\gamma_{111}$ and $\gamma_{001},{ }^{60}$ as well as the growth velocities along $<111>$ and $<001>$ directions of the crystal.${ }^{61}$ Thermodynamically the $s / l$ ratio is a constant irrespective of the supporting substrate, and it is related to the surface energies via $\frac{\gamma_{111}}{\gamma_{001}}=$ $\frac{1}{\sqrt{3}}+\frac{s}{2 l}$. However, the growth velocities of the crystallographic facets are differently influenced by the local environment, which, when outside UHV, can involve hydrocarbons in air, CO gas, and other adsorbates. $^{62}$

In Fig. 5a, the $s / l$ ratio on the three $\mathrm{SrTiO}_{3}$ substrates is plotted against crystal height $h$. Again, only those crystals grown at $>300{ }^{\circ} \mathrm{C}$ were measured, which are reasonably close to their equilibrium shapes. Apart from the flat crystals on $\mathrm{SrTiO}_{3}(001)-(2 \times 1)$ for which $h<4 \mathrm{~nm}$, the $s / l$ ratio is a constant, with values of $0.15 \pm 0.07$ on $\mathrm{SrTiO}_{3}(001)-(2 \times 1), 0.28 \pm 0.06$ on $\mathrm{SrTiO}_{3}(001)-c(4 \times 2)$, and $0.46 \pm 0.09$ on $\mathrm{SrTiO}_{3}(111)-(4 \times 4)+(6 \times 6)$. Since the gold crystals were grown and characterized in UHV, their different $s / l$ ratios on different substrates are unexpected.

Among all three substrates, $\mathrm{SrTiO}_{3}(111)-(4 \times 4)+(6 \times 6)$ gives rise to the largest $s / l$ ratio. In the STM images in Fig. 1d, the gold crystal top facets are close to regular hexagons. Consistently, Fig. 5e,f shows 
two SEM images (top and tilted views) of gold crystals on $\mathrm{SrTiO}_{3}(111)-(4 \times 4)+(6 \times 6)$, in which the large crystals were obtained following a post anneal at $600{ }^{\circ} \mathrm{C}$ for $5 \mathrm{~h}$. Their $s / l$ ratio is measured to be $0.55 \pm 0.02$, within the range of values obtained from STM images, $0.46 \pm 0.09$.
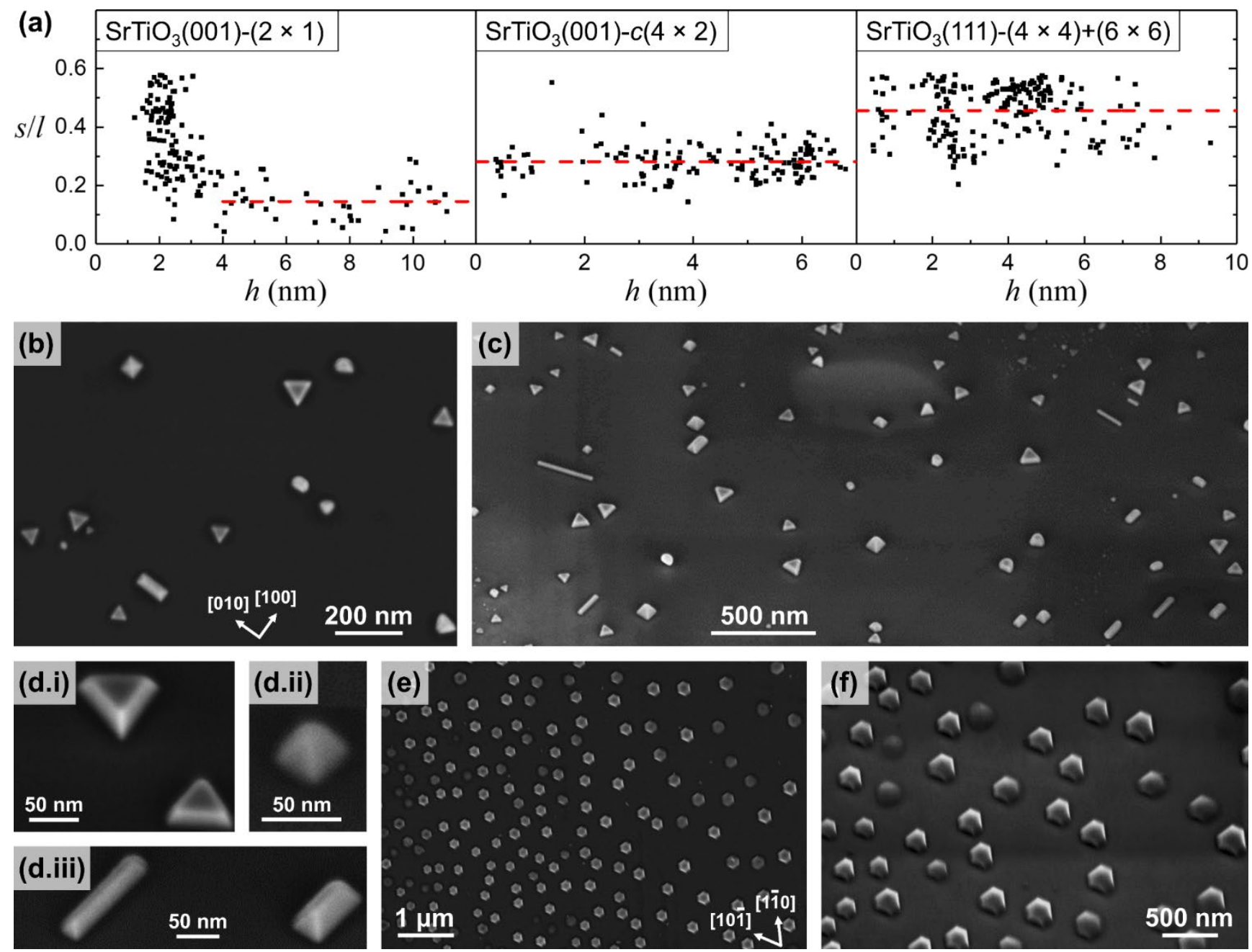

Fig. 5: $s / l$ ratio of gold crystals and SEM images. (a) $s / l$ ratio measured from STM images plotted against crystal height on $\mathrm{SrTiO}_{3}(001)-(2 \times 1), \mathrm{SrTiO}_{3}(001)-c(4 \times 2)$, and $\mathrm{SrTiO}_{3}(111)-(4 \times 4)+(6 \times 6)$. (b-d) $\mathrm{SEM}$ images of gold crystals on $\mathrm{SrTiO}_{3}(001)-(2 \times 1)$ : (b) top view, (c) tilted view $\left(40^{\circ}\right)$, and (d) high-magnification images of the three typical crystal shapes: (d.i) triangles, (d.ii) pyramids, and (d.iii) huts. (e) Top view and (f) tilted view $\left(40^{\circ}\right)$ of gold crystals on $\mathrm{SrTiO}_{3}(111)-(4 \times 4)+(6 \times 6)$.

On the other hand, the relatively high gold crystals $(h>4 \mathrm{~nm})$ on $\mathrm{SrTiO}_{3}(001)-(2 \times 1)$ have very small $\{001\}$ facets that are barely visible. The crystal top facets look like triangles without truncation, e.g., the highest (brightest) four crystals in Fig. 1b. This is also confirmed by the triangular crystals in Fig. 
5b-d, which are SEM images of some large crystals on $\mathrm{SrTiO}_{3}(001)-(2 \times 1)$ prepared by a post anneal at $500{ }^{\circ} \mathrm{C}$ for $1 \mathrm{~h} . \mathrm{On} \mathrm{SrTiO}_{3}(001)-(2 \times 1)$, three crystal shapes are observed: "triangles", "pyramids", and "huts", as shown in the three high-magnification images in Fig. 5d.i-iii, in this order. They are all produced by different orientations of the Wulff shape of gold, and their crystallographic interfaces with the substrates are (111), (001), and (110), respectively. ${ }^{24}$ In Fig. 5b-d, the triangles and pyramids do not show any visible $\{001\}$ facets and the huts are trying to grow infinitely long to minimize the proportion of $\{001\}$ facets. These are in good agreement with the $s / l$ plot for $\mathrm{SrTiO}_{3}(001)-(2 \times 1)($ Fig. $5 \mathrm{a})$, in which the equilibrium $s / l$ ratio $=0.15 \pm 0.07$ at large crystal heights.

As an aside, thermodynamically the three crystal shapes (triangles, pyramids, and huts) are not expected to coexist on the same substrate. Previously we explained that the lowest energy morphology depends on $\gamma^{*}=\gamma_{\mathrm{i}}-\gamma_{\mathrm{s} .}{ }^{24}$ For example, the stable structure of fcc Pd crystals was reported to be the hut shape on $\mathrm{SrTiO}_{3}(001)-(2 \times 1)$ and the pyramid shape on $\mathrm{SrTiO}_{3}(001)-c(4 \times 2) \cdot{ }^{24} \mathrm{In}$ our system, the truncated triangular crystals are identified as the thermodynamically favored shape on all three substrates. It is the only shape observed for small crystals under STM (Fig. 1) and the most frequently observed shape for large crystals under SEM (Fig. 5b-f). The pyramids and huts may nucleate randomly and become stuck in these metastable orientations due to kinetics. ${ }^{43}$

Interestingly, the flat crystals $(h<4 \mathrm{~nm})$ on $\mathrm{SrTiO}_{3}(001)-(2 \times 1)$ have a decreasing $s / l$ ratio with increasing crystal height, from $\frac{1}{\sqrt{3}}(\approx 0.58)$ to the equilibrium value of 0.15 (Fig. 5a). $s / l$ equals $\frac{1}{\sqrt{3}}$ when the crystal top facet is a regular hexagon. We believe that the varying $s / l$ ratio of the flat crystals can be attributed to the dewetting phenomenon of gold monolayers, which only exist on $\mathrm{SrTiO}_{3}(001)$ $(2 \times 1)$. We notice that when the monolayers are annealed at $300-400{ }^{\circ} \mathrm{C}$, they tend to develop into regular hexagons, possibly in preparation for their conversion into crystals. The gold atoms may become 
close-packed as a hexagonal monolayer, where its six sides are crystallographically equivalent, because the edges have not yet differentiated themselves into $\{111\}$ and $\{001\}$ side facets. During the dewetting, three alternating sides of the hexagon develop into $\{111\}$ side facets and the other three into $\{001\}$. In this process, its top facet turns from a regular hexagon into a truncated triangle. In Fig. 1a.ii,b.ii, the crystal heights are labeled below each crystal and we can see that the higher a crystal is, the more "pointed" its top facet is, i.e., smaller $\{001\}$ side facets. In Fig. 1a the flattest crystal $(h=1.72 \mathrm{~nm})$ even has a curved edge, which may be a residual feature from an irregularly shaped monolayer.

It is also interesting that the flattest crystals observed on $\mathrm{SrTiO}_{3}(001)-(2 \times 1)$ consist of a minimum of five atomic layers, whereas on $\mathrm{SrTiO}_{3}(001)-c(4 \times 2)$ and $\mathrm{SrTiO}_{3}(111)-(4 \times 4)+(6 \times 6), 2-4$-layer crystals have been found. Accordingly, the data points for $\mathrm{SrTiO}_{3}(001)-(2 \times 1)$ in Fig. 4a and Fig. 5a only start from $h \sim 1.2 \mathrm{~nm}$. We speculate that the monolayers are the nucleation shape of small gold islands on $\mathrm{SrTiO}_{3}(001)-(2 \times 1)$, which represent a local minimum in energy. Under diffusion-limited conditions, there is a growth barrier to 3D crystals. This activation barrier appears to require the assembly of five atomic layers at a minimum.

Now we explain the possible origin of the different $s / l$ ratios of gold crystals on the three $\mathrm{SrTiO}_{3}$ substrates. In our study, the gold crystals were prepared in a UHV environment nearly free of gaseous adsorbates, so their different $s / l$ ratios have to be attributed to their interaction with the substrates. Previously, when gold crystals were studied in UHV, e.g., on $\mathrm{CeO}_{2}(001)$ and $\mathrm{SrTiO}_{3}(001)-(2 \times 1)$ substrates, ${ }^{23,28}$ they grew into the truncated triangular shape as well. However, in these studies the $s / l$ ratio of the crystals was not specifically analyzed. We have not reached a firm conclusion of the mechanism by which the substrates influence the $s / l$ ratio, but we discuss a few possibilities below.

Firstly, the three $\mathrm{SrTiO}_{3}$ substrates certainly interact with the gold crystals differently at the interface. 
For example, the interfacial strain is calculated to be $1.5 \%$ on $\mathrm{SrTiO}_{3}(001)$ and $4.4 \%$ on $\mathrm{SrTiO}_{3}(111)$. However, we propose that the interfacial interaction itself is not the direct cause of the different $s / l$ ratios. Taking the $\mathrm{SrTiO}_{3}(001)-(2 \times 1)$ substrate as an example: any interfacial effect may possibly cause the $\{001\}$ side facets of a truncated triangular gold crystal to shrink, but the interface should not affect the (001) top facet of a pyramidal crystal. However, the latter is also seen to be minimized (Fig. 5b-d).

The second possibility is the encapsulation of gold crystals by an oxide layer from the substrate, known as the strong metal-support interaction (SMSI). ${ }^{63-65}$ Encapsulation by an oxide layer is driven by the reduction in the metal facet energies. One would expect the $\{111\}$ and $\{001\}$ gold facets to undergo different degrees of energy reduction. Hence, this would influence the $s / l$ ratio. However, metal particles encapsulated by $\mathrm{TiO}_{x}$ layers usually exhibit superstructures or moiré patterns under STM observation, ${ }^{63,65,66}$ which are not seen on our gold crystals. For example, when fcc Pd crystals with a (111) base are grown on nanostructured $\mathrm{SrTiO}_{3}(001)$, they exhibit two clear moiré patterns: the "wagon wheel" and the "hexagonal superstructure". ${ }^{63}$ When (111)-based Pd crystals are supported on $\mathrm{TiO}_{2}(110)$ substrates, two superstructures named "pinwheels" and "zigzags" are observed. ${ }^{65,66}$ We have not found publications on $\mathrm{TiO}_{x}$-encapsulated gold crystals, but superstructures formed by $\mathrm{TiO}_{x}$ ultrathin films on an $\mathrm{Au}(111)$ surface have been reported: "honeycomb", "pinwheel", and triangular islands. ${ }^{67}$ In our system, the absence of any overlayer structures on the gold crystal top facets means that there is no evidence for $\mathrm{TiO}_{x}$ encapsulation.

A third possibility is the presence of surfactants originating from the $\mathrm{SrTiO}_{3}$ substrates. The $\mathrm{SrTiO}_{3}(001)-(2 \times 1)$ surface is hydroxylated, i.e., it is terminated with $-\mathrm{OH}$ groups. ${ }^{38}$ The oxygen is part of $\mathrm{TiO}_{5}$ or $\mathrm{TiO}_{6}$ groups, but the hydrogen can desorb from the substrate so that the gold crystals grown on this surface may become hydrogen-terminated. The hydrogen may preferentially bind to the $\{111\}$ 
facets compared with the $\{001\}$ facets, inhibiting the growth along $<111>$ directions. Another way to depict this scenario is that the hydrogen surfactant reduces the facet energy of $\{111\}$ to a greater extent than that of $\{001\}$, promoting the growth of $\{111\}$ facets. Nevertheless, it is not obvious to us what surfactants might arise from the other two substrates, $\mathrm{SrTiO}_{3}(001)-c(4 \times 2)$ and $\mathrm{SrTiO}_{3}(111)-(4 \times 4)+(6$ $\times 6$ ). According to previous studies, these two surfaces are both based on Ti-centered polyhedral building blocks $\left(\mathrm{TiO}_{4}, \mathrm{TiO}_{5}\right.$, and $\left.\mathrm{TiO}_{6}\right)$ in the top few layers and are not terminated with any easily dissociable species..$^{39,42}$

Previous reports on gold crystal shapes are mostly from crystals grown in solution, e.g., using the polyol synthetic method. ${ }^{11-15}$ During solvent-based growth, the ratio between the $\{111\}$ and $\{001\}$ surface areas $\left(A_{111} / A_{001}\right)$ is heavily influenced by the selective adsorption of surfactants on certain crystallographic planes. ${ }^{12-16,68}$ This gives rise to crystal shapes that range from octahedra (purely $\{111\}$ terminated) to cubes (purely $\{001\}$-terminated). ${ }^{12-15,69}$ For example, it was discovered that higher concentrations of poly(vinyl pyrrolidone) (PVP) and poly(diallyldimethylammonium) chloride (PDDA) encourage the formation of a higher proportion of $\{111\}$ facets. ${ }^{12-14}$ On the other hand, $\{001\}$ facets of gold crystals were reported to be stabilized by silver ions and cetyltrimethylammonium bromide (CTAB). ${ }^{12,15,16,68}$

\subsection{Wulff and growth shapes}

The SEM images of gold crystals in Fig. 5c,d,f were taken with the samples tilted at $40^{\circ}$ and there is no evidence of any reentrant facets. According to the geometry of the Wulff crystal (Fig. 3a), reentrant facets should appear if the crystal height is greater than $\sqrt{\frac{2}{3}} l$. We know from STM images that $75 \%$ of 
our crystals grown at $>300{ }^{\circ} \mathrm{C}$ satisfy the condition that $h>\sqrt{\frac{2}{3}} l ; 85 \%$ of our crystals grown at $>$ $350{ }^{\circ} \mathrm{C}$ satisfy this condition. Provided that the gold crystals in Fig. $5 \mathrm{~b}-\mathrm{f}$ were treated at $500{ }^{\circ} \mathrm{C}$ and above, most of the hexagonal or triangular crystals would show reentrant facets if they followed the Wulff shape. However, when kinetics play a key part, an enhancement factor is added to the growth velocity of the reentrant facets. ${ }^{61}$ An analogy is that in heterogeneous nucleation, the nucleation energy is lower on a notched surface than on a smooth surface. The bottom of a reentrant facet acts as such a notch and the atoms attaching to such a site form additional bonds with the substrate. Therefore, for an equilibrium crystal with the shape in Fig. 3a, the attachment energy of additional gold atoms to the reentrant $\{111\}$ facets would be lower than that to the top (111) facet. Moreover, most gold atoms arrive at a crystal sideways from the substrate surface due to Ostwald ripening rather than from the top during the gold evaporation. Hence reentrant facets typically grow out.

Now we discuss the geometries and energies of the Wulff and growth shapes. When $\frac{\gamma_{001}}{\gamma_{111}} \geq \sqrt{3}$, the Wulff shape of a gold crystal does not have $\{001\}$ facets and is an octahedron (Fig. 6a). The first row of drawings in Fig. 6a shows how the Wulff shape (yellow) can be cut to produce the three substratesupported crystal shapes: "triangle", "pyramid", and "hut" (orange). The triangular and pyramidal crystals both have reentrant facets. When these reentrant facets grow out, their corresponding growth shapes result, as illustrated in the second row in Fig. 6a. Note that the hut shape has four $\{111\}$ end facets, two on each end, and they are perpendicular to the substrate. Therefore, the hut does not have reentrant facets (with a contact angle of $>90^{\circ}$ with the substrate), and hence its growth shape is the same as its Wulff shape. When $1<\frac{\gamma_{001}}{\gamma_{111}}<\sqrt{3},\{001\}$ and $\{111\}$ facets of the gold crystals coexist and the Wulff shape is a truncated octahedron (Fig. 6b). Fig. 6b shows a similar table of sketches to Fig. 6a. When $\{001\}$ facets are present, the "triangular" crystal has a truncated triangular top facet, and is therefore called a "hexagon" for simplicity. 


Triangle Pyramid
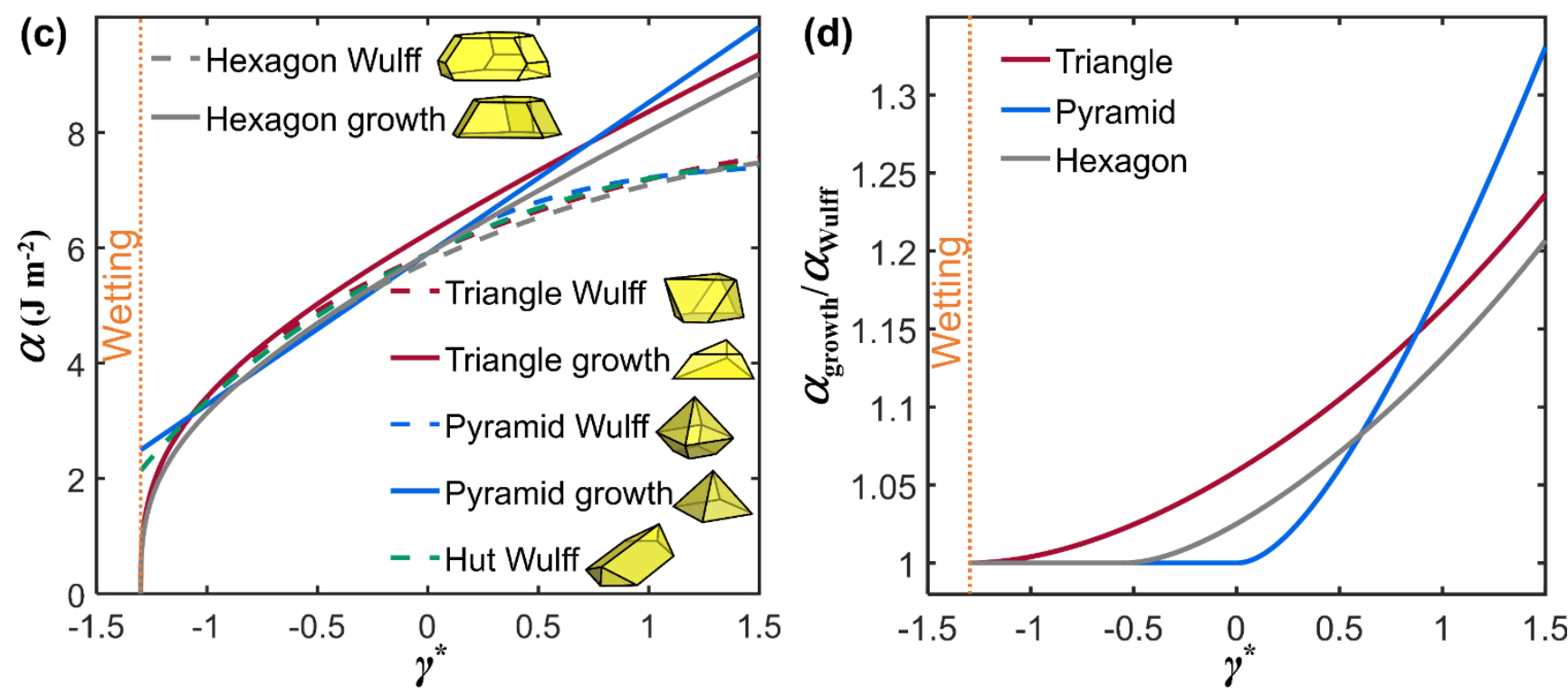

Fig. 6: Geometries and energies of the Wulff and growth shapes of gold crystals. (a) Geometries of \{111\}terminated gold crystals. The first row shows the Wulff shapes (yellow) being cut to produce triangular, pyramidal, and hut crystals (orange). Their corresponding growth shapes are drawn below them in the second row. (b) Wulff and growth shapes of gold crystals with both $\{111\}$ and $\{001\}$ facets. With $\{001\}$ facets present, the triangular crystal has a truncated triangular top facet and is called a "hexagon" for simplicity. (c) Plots of $\alpha$ versus $\gamma^{*}$ for the Wulff and growth shapes of the triangle, pyramid, hut, and hexagon. (d) Plots of growth-to-Wulff $\alpha$ ratios for the triangle, pyramid, and hexagon. In (c,d), facet energies of $\gamma_{111}=1.3 \mathrm{~J} \mathrm{~m}^{-2}$ and $\gamma_{001}=1.6 \mathrm{~J} \mathrm{~m}^{-2}$ are used for gold; ${ }^{70}$ gold wets the substrate when $\gamma^{*}<-\gamma_{111}=$ $-1.3 \mathrm{~J} \mathrm{~m}^{-2}$.

As mentioned in section 3.2, the total energy of a substrate-supported crystal consists of the formation energy, the surface/interface energies, and the strain energy. ${ }^{29,30,50}$ For a crystal with a given volume, its Wulff and growth shapes have the same formation energy. Their surface/interface energies and strain energies will differ, though we do not have sufficient information to accurately calculate their strain 
energies. However, we can estimate the surface/interface energies of the three crystal shapes: the Wulff and growth shapes of triangles/hexagons, the Wulff and growth shapes of pyramids, and the Wulff shape of huts. The total surface/interface energy of a nanocrystal can be parameterized as $E=\alpha_{\text {shape }} V^{\frac{2}{3}}, 24,50$ where $V$ is the crystal volume and $\alpha_{\text {shape }}$ is discussed below for the two cases where there either are, or are not $\{001\}$ facets present.

Without the $\{001\}$ facets, i.e., when $\frac{\gamma_{001}}{\gamma_{111}} \geq \sqrt{3}$, the following relationships can be derived:

$$
\begin{gathered}
\alpha_{\text {triangle Wulff }}=3^{\frac{7}{6}} \cdot 2^{-\frac{2}{3}}\left(8 \gamma_{111}^{3}+9 \gamma_{111}^{2} \gamma^{*}-\gamma^{* 3}\right)^{\frac{1}{3}} \\
\alpha_{\text {triangle growth }}=\frac{3}{2}\left[\sqrt{3}\left(19 \gamma_{111}^{3}+27 \gamma_{111}^{2} \gamma^{*}+9 \gamma_{111} \gamma^{* 2}+\gamma^{* 3}\right)\right]^{\frac{1}{3}} \\
\alpha_{\text {pyramid Wulff }}=3^{\frac{2}{3}}\left(6 \sqrt{3} \gamma_{111}^{3}+18 \gamma_{111}^{2} \gamma^{*}-6 \sqrt{3} \gamma_{111} \gamma^{* 2}+2 \gamma^{* 3}\right)^{\frac{1}{3}} \\
\alpha_{\text {pyramid growth }}=18^{\frac{1}{3}}\left(\sqrt{3} \gamma_{111}+\gamma^{*}\right) \\
\alpha_{\text {hut Wulff }}=3^{\frac{2}{3}} \cdot 2^{-\frac{1}{6}}\left(\sqrt{6} \gamma_{111}-\gamma^{*}\right)^{\frac{1}{3}}\left(\sqrt{6} \gamma_{111}+2 \gamma^{*}\right)^{\frac{2}{3}}
\end{gathered}
$$

The $\alpha_{\text {shape }}$ formulae can also be derived for crystals when $\{001\}$ facets are present, i.e., when $1<\frac{\gamma_{001}}{\gamma_{111}}$ $<\sqrt{3}$. We only focus on the (111)-based crystals, which are the experimentally observed equilibrium shape, and they are called "hexagons" with $\{001\}$ facets present. Now,

$$
\begin{gathered}
\alpha_{\text {hexagon Wulff }}=3 \cdot 2^{-\frac{2}{3}}\left(8 \gamma_{001}^{3}-24 \sqrt{3} \gamma_{001}^{2} \gamma_{111}+72 \gamma_{001} \gamma_{111}^{2}-16 \sqrt{3} \gamma_{111}^{3}+9 \sqrt{3} \gamma_{111}^{2} \gamma^{*}-\sqrt{3} \gamma^{* 3}\right)^{\frac{1}{3}} \\
\alpha_{\text {hexagon growth }}=\frac{3}{2}\left\{\left(\gamma_{111}+\gamma^{*}\right)\left[72 \gamma_{001} \gamma_{111}-12 \sqrt{3} \gamma_{001}^{2}+\sqrt{3}\left(\gamma^{* 2}+8 \gamma_{111} \gamma^{*}-17 \gamma_{111}^{2}\right)\right]\right\}^{\frac{1}{3}}
\end{gathered}
$$

$\alpha_{\text {shape }}$ is plotted as a function of $\gamma^{*}$ in Fig. $6 \mathrm{c}$ using the theoretical values of $\gamma_{111}=1.3 \mathrm{~J} \mathrm{~m}^{-2}$ and $\gamma_{001}=$ $1.6 \mathrm{~J} \mathrm{~m}^{-2} \cdot{ }^{70}$ The corresponding shapes are sketched in the legend. For the triangular, pyramidal, and 
hexagonal crystals, their Wulff shape is always lower in energy than their growth shape. The growth/Wulff energy discrepancy increases with increasing $\gamma^{*}$, as shown in the plot of their $\alpha$ ratios in Fig. $6 \mathrm{~d}$. At a practical value of $\gamma^{*}$ such as $-0.5 \mathrm{~J} \mathrm{~m}^{-2}$, there is a reasonably small discrepancy between the surface/interface energies of the Wulff and growth shapes $(<3 \%)$. This is a condition that helps the growth shapes to survive.

For the pyramid, its Wulff shape is the same as the growth shape when the Wulff point of the crystal (or the midpoint of the Wulff octahedron) is below the substrate surface. Therefore their $\alpha$ plots in Fig. 6c (two blue lines) only start to diverge when $\gamma^{*}>0$. For the hexagon, the energies of its Wulff and growth shapes only start to diverge at $\gamma^{*}=-0.5 \mathrm{~J} \mathrm{~m}^{-2}$ (two gray lines in Fig. 6c), because for any lower value of $\gamma^{*}$ the Wulff crystal does not yet have reentrant facets using values of $\gamma_{111}=1.3 \mathrm{~J} \mathrm{~m}^{-2}$ and $\gamma_{001}=1.6 \mathrm{~J} \mathrm{~m}^{-}$ 2 .

If we define $\gamma_{\mathrm{m}}$ as the surface energy of a gold monolayer per unit area and $\gamma_{\mathrm{im}}$ as the monolayersubstrate interfacial energy, the condition for wetting is $\gamma_{\mathrm{m}}+\gamma_{\mathrm{im}}-\gamma_{\mathrm{s}}<0$ (if the edge energy of monolayers is ignored). Since $\gamma^{*}=\gamma_{\mathrm{im}}-\gamma_{\mathrm{s}}$, the equation above can be rearranged as $\gamma^{*}<-\gamma_{\mathrm{m}}$. We do not know the value of $\gamma_{\mathrm{m}}$ but in a monolayer, the closest packing possible of the gold atoms corresponds to an $\mathrm{Au}(111)$ structure, with a surface energy of $\gamma_{111}=1.3 \mathrm{~J} \mathrm{~m}^{-2}$. Therefore in Fig. $6 \mathrm{c}$, d, when $\gamma^{*}<-1.3 \mathrm{~J} \mathrm{~m}^{-2}$ gold wets the substrate and the $\alpha$ plots of the crystals are only physically meaningful outside this regime.

The energy calculations presented in Fig. 6c,d are only a qualitative comparison between the Wulff and growth shapes, because they do not take into account the effects of the crystal-substrate interfacial strain (section 3.2) and the chemical environments (section 3.3). Because of the latter, the $A_{111} / A_{001}$ ratio of experimentally observed gold crystals is affected by surfactant adsorption on their $\{111\}$ and $\{001\}$ facets. It is therefore not a true reflection of the pristine gold facet energies $\left(\gamma_{111}\right.$ and $\left.\gamma_{001}\right)$. Hence, our 
use of $\gamma_{111}=1.3 \mathrm{~J} \mathrm{~m}^{-2}$ and $\gamma_{001}=1.6 \mathrm{~J} \mathrm{~m}^{-2}$ is only an approximation.

Additionally, we would like to comment that the growth shape of the triangular crystal in Fig. 6a has a truncated tetrahedral shape and is terminated with $\{111\}$ planes only. If such a crystal grew into a full tetrahedron, geometrically it would be the constituent part of MTPs, e.g., decahedra and icosahedra (though these structures are not "built up" by the tetrahedra, but rather form by twinning). Gold crystals with the truncated tetrahedral shape have been previously observed as free-standing crystals prepared in solution, ${ }^{12,13}$ but the authors did not relate them to the Wulff shape. Here, we understand that this shape is a deviation from the $\{111\}$-terminated Wulff shape, i.e., an octahedron cut by the substrate, upon which the reentrant facets grow out. It is difficult to explain why in previous studies the freestanding gold crystals also ended up with this growth shape, ${ }^{12,13}$ as there were no substrates to encourage the growing out of reentrant facets. It is possibly attributable to their very rapid growth and the small energy difference between the Wulff and growth shapes. Alternatively, some of the crystals might have attached to the test tube walls during growth.

Finally, on some substrate-supported gold nanocrystals, reentrant facets have been observed previously, e.g., on $\mathrm{CeO}_{2}$ and $\mathrm{TiO}_{2}$ substrates. ${ }^{23,35,71-74}$ These gold crystals adopt the same equilibrium shape as that in our work, with an $\mathrm{Au}(111)$ interface with the substrate. They were characterized by scanning transmission electron microscopy (STEM) and typically have a lateral size of $\sim 5 \mathrm{~nm}$, cf. our gold crystals in the SEM images (Fig. 5b-f), which are 50-300 nm laterally. In some of the previous studies, a deposition-precipitation method was used to prepare the gold crystals. ${ }^{23,71,72} \mathrm{~A}$ few grams of the oxide particles $\left(\mathrm{CeO}_{2}\right.$ and $\left.\mathrm{TiO}_{2}\right)$ were dispersed into an aqueous solution of $\mathrm{HAuCl}_{4}$. Following centrifugation, washing, and drying, the samples were calcinated at $300-400{ }^{\circ} \mathrm{C}$ in air, which led to the decomposition of the $\mathrm{Au}(\mathrm{III})$ complexes into gold metal particles. This is in essence a solvent-based growth method, 
and the presence of gold reentrant facets is not surprising. ${ }^{61}$

However, in some other studies gold was deposited onto $\mathrm{TiO}_{2}$ substrates using an electron-beam evaporator at a rate of $0.33 \AA \mathrm{min}^{-1}$ in vacuum..$^{35,73,74}$ The sample was then annealed at $400-700{ }^{\circ} \mathrm{C}$ to promote the formation of equilibrium crystal shapes. This protocol is similar to ours, where we deposited gold at an even lower rate $\left(\sim 0.06 \AA \mathrm{min}^{-1}\right)$ and annealed the crystals at similar temperatures $\left(500-600{ }^{\circ} \mathrm{C}\right.$ ) before taking the SEM images (Fig. 5b-f). We believe that the presence of reentrant facets on their gold crystals is due to their smaller crystal sizes. When the diameter of a gold particle is smaller than $\sim 20 \mathrm{~nm}$, their melting point drops below that of bulk gold $\left(1064^{\circ} \mathrm{C}\right)$, and it is calculated to be $\sim 700{ }^{\circ} \mathrm{C}$ for a $5 \mathrm{~nm}$-diameter particle. ${ }^{75,76}$ Therefore, in Ref. ${ }^{35,73,74}$ the gold crystals were post-annealed at temperatures close to their melting point. They may have become molten hemispheres during the thermal treatment, which, when cooled down, are expected to reach the thermodynamically optimized shapes, i.e., the Wulff shapes with reentrant facets present.

\section{Conclusions}

In conclusion, the gold crystal shapes are strongly influenced by their interaction with the $\mathrm{SrTiO}_{3}$ substrates: $\mathrm{SrTiO}_{3}(001)-(2 \times 1), \mathrm{SrTiO}_{3}(001)-c(4 \times 2)$, and $\mathrm{SrTiO}_{3}(111)-(4 \times 4)+(6 \times 6)$. The crystal shape is not self-similar between different crystal sizes. The $h / l$ ratio increases with the crystal height $h$ on all three substrates, because crystal heightening partially releases the interfacial strain caused by the lattice mismatch. The $s / l$ ratio, which is a direct measure of $A_{111} / A_{001}$, is found to differ on the three substrates. This may be due to the selective adsorption of surfactants on the $\{111\}$ and $\{001\}$ gold facets. One possible surfactant is chemisorbed hydrogen on the hydroxylated $\mathrm{SrTiO}_{3}(001)-(2 \times 1)$ surface. The 
flat crystals on $\mathrm{SrTiO}_{3}(001)-(2 \times 1)(h<4 \mathrm{~nm})$ are an exception, where their $s / l$ ratio decays from $\frac{1}{\sqrt{3}}$ towards the equilibrium value of 0.15 with increasing $h$. This is likely to relate to the dewetting process from monolayers. Also, the crystal reentrant facets are not observed because these concave facets typically grow out due to kinetics. These growth shapes are slightly higher in energy than their thermodynamic Wulff shape, and the energy discrepancy is greater at larger values of $\gamma^{*}$. This study demonstrates the influence of the detailed nature of oxide substrate surfaces on the shape of epitaxial gold nanocrystals, which is a phenomenon critical to the rational tuning of their catalytic and optical properties. Further research in this area would be important with respect to realizing their full potential in catalysis, biological sensing, and photonics.

\section{Conflicts of interest}

There are no conflicts to declare.

\section{Acknowledgements}

This work was supported by the EPSRC grant EP/M015173/1. The authors would like to thank Chris Spencer (JEOL U.K.) for technical support.

\section{References}

1 M. Haruta, T. Kobayashi, H. Sano and N. Yamada, Chem. Lett., 1987, 405-408. 
M. S. Chen and D. W. Goodman, Catal. Today, 2006, 111, 22-33.

B. Hvolbæk, T. V. W. Janssens, B. S. Clausen, H. Falsig, C. H. Christensen and J. K. Nørskov, Nano Today, 2007, 2, 14-18.

Y. G. Wang, D. Mei, V. A. Glezakou, J. Li and R. Rousseau, Nat. Commun., 2015, 6, 6511.

N. M. Andoy, X. Zhou, E. Choudhary, H. Shen, G. Liu and P. Chen, J. Am. Chem. Soc., 2013, 135, 1845-1852.

N. Tian, Z. Zhou, S. Sun, Y. Ding and Z. L. Wang, Science, 2007, 316, 732-735.

M. Boronat and A. Corma, Dalt. Trans., 2010, 39, 8538-8546.

J. N. Anker, W. P. Hall, O. Lyanders, N. C. Shah, J. Zhao and R. P. Van Duyne, Nat. Mater., 2008, 6, 442-453.

K. M. Mayer and J. H. Hafner, Chem. Rev., 2011, 111, 3828-3857. E. Ringe, M. R. Langille, K. Sohn, J. Zhang, J. Huan
Marks, J. Phys. Chem. Lett., 2012, 3, 1479-1483.

F. Kim, S. Connor, H. Song, T. Kuykendall and P. Yang, Angew. Chemie Int. Ed., 2004, 43, 36733677.

D. Seo, C. I. Yoo, I. S. Chung, S. M. Park, S. Ryu and H. Song, J. Phys. Chem. C, 2008, 112, 2469-2475.

C. Li, K. L. Shuford, M. Chen, E. J. Lee and S. O. Cho, ACS Nano, 2008, 2, 1760-1769.

D. Seo, C. P. Ji and H. Song, J. Am. Chem. Soc., 2006, 128, 14863-14870.

K. Sohn, F. Kim, K. C. Pradel, J. Wu, Y. Peng, F. Zhou and J. Huang, ACS Nano, 2009, 3, 21912198.

M. Mulvihill, A. Tao, K. Benjauthrit, J. Arnold and P. Yang, Angew. Chemie Int. Ed., 2008, 47, 6456-6460.

(18)

A. S. Barnard, N. P. Young, A. I. Kirkland, M. A. van Huis and H. Xu, ACS Nano, 2009, 3, 14311436.

W. L. Winterbottom, Acta Metall., 1967, 15, 303-310.

R. Kaishew, Arbeitstagung Festkörper Physik, Dresden, 1952.

K. H. Hansen, T. Worren, S. Stempel, E. Lægsgaard, M. Bäumer, H.-J. Freund, F. Besenbacher and I. Stensgaard, Phys. Rev. Lett., 2002, 83, 4120-4123.

J. A. Enterkin, K. R. Poeppelmeier and L. D. Marks, Nano Lett., 2011, 11, 993-997.

Y. Lin, Z. Wu, J. Wen, K. Ding, X. Yang, K. R. Poeppelmeier and L. D. Marks, Nano Lett., 2015, 15, 5375-5381.

F. Silly and M. R. Castell, Phys. Rev. Lett., 2005, 94, 046103.

F. Silly and M. R. Castell, Appl. Phys. Lett., 2005, 87, 063106.

J. Sun, C. Wu, F. Silly, A. A. K. Koo, F. Dillon, N. Grobert and M. R. Castell, Chem. Commun., 2013, 49, 3748-3750.

27 F. Silly and M. R. Castell, Appl. Phys. Lett., 2005, 87, 053106.

28 F. Silly and M. R. Castell, Phys. Rev. Lett., 2006, 96, 086104.

29 P. Müller and R. Kern, Surf. Sci., 2000, 457, 229-253.

30 V. A. Shchukin and D. Bimberg, Rev. Mod. Phys., 2002, 71, 1125-1171.

31 P. Müller and R. Kern, J. Cryst. Growth, 1998, 193, 257-270.

32 P. Müller and R. Kern, Microsc. Microanal. Microstruct., 1997, 8, 229-238.

33 N. Mammen, S. Narasimhan and S. de Gironcoli, J. Am. Chem. Soc., 2011, 133, 2801-2803.

34 R. Si and M. Flytzani-Stephanopoulos, Angew. Chemie Int. Ed., 2008, 47, 2884-2887.

35 S. Sivaramakrishnan, J. Wen, M. E. Scarpelli, B. J. Pierce and J.-M. Zuo, Phys. Rev. B, 2010, 82, 
195421.

N. Lopez, J. K. Nørskov, T. V. W. Janssens, A. Carlsson, A. Puig-Molina, B. S. Clausen and J. D. Grunwaldt, J. Catal., 2004, 225, 86-94.

M. R. Castell, Surf. Sci., 2002, 505, 1-13.

A. E. Becerra-Toledo, M. R. Castell and L. D. Marks, Surf. Sci., 2012, 606, 762-765.

A. E. Becerra-Toledo, M. S. J. Marshall, M. R. Castell and L. D. Marks, J. Chem. Phys., 2012, 136, 214701.

M. R. Castell, Surf. Sci., 2002, 516, 33-42.

B. C. Russell and M. R. Castell, J. Phys. Chem. C, 2008, 112, 6538-6545.

L. D. Marks, A. N. Chiaramonti, S. ur Rahman and M. R. Castell, Phys. Rev. Lett., 2015, 114, 226101.

43 L. D. Marks and L. Peng, J. Phys. Condens. Matter, 2016, 28, 53001.

44 L. Jones, S. Wang, X. Hu, S. ur Rahman and M. R. Castell, Adv. Struct. Chem. Imaging, 2018, 4, 7.

45 L. Jones, H. Yang, T. J. Pennycook, M. S. J. Marshall, S. Van Aert, N. D. Browning, M. R. Castell and P. D. Nellist, Adv. Struct. Chem. Imaging, 2015, 1, 8.

I. Horcas, R. Fernández, J. M. Gómez-Rodríguez, J. Colchero, J. Gómez-Herrero and A. M. Baro, Rev. Sci. Instrum., 2007, 78, 013705.

F. Silly and M. R. Castell, Appl. Phys. Lett., 2005, 87, 213107.

L. D. Marks, Philos. Mag. A, 1984, 49, 81-93.

Y. Furuhata, E. Nakamura and E. Sawaguchi, Landolt-Börnstein, Group III, 1969, vol. 16.

G. Medeiros-Ribeiro, A. M. Bratkovski, T. I. Kamins, D. A. A. Ohlberg and R. S. Williams, Science, 1998, 279, 353-355.

I. S. Grigoriev, E. Z. Meilikhov and A. A. Radzig, Handbook of Physical Quantities, CRC Press, 1996.

52 D. de Ligny and P. Richet, Phys. Rev. B, 1996, 53, 3013-3022.

53 O. Warschkow, M. Asta, N. Erdman, K. R. Poeppelmeier, D. E. Ellis and L. D. Marks, Surf. Sci., 2004, 573, 446-456.

54 P. F. Kelly, Properties of Materials, CRC Press, 2014.

55 O. Bernard, M. Andrieux, S. Poissonnet and A. M. Huntz, J. Eur. Ceram. Soc., 2004, 24, 763773.

J. B. Wachtman, M. L. Wheat and S. Marzullo, J. Res. Natl. Bur. Stand. Sect. A, 1963, 67A, 193209.

57 M. Hammar, F. K. LeGoues, J. Tersoff, M. C. Reuter and R. M. Tromp, Surf. Sci., 1996, 349, $129-144$.

58 M. Ahmadi, F. Behafarid and B. R. Cuenya, Nanoscale, 2016, 8, 11635-11641.

59 Z. Zhang, L. Li and J. C. Yang, J. Phys. Chem. C, 2013, 117, 21407-21412.

60 M. R. Castell, Phys. Rev. B, 2003, 68, 235411.

61 E. Ringe, R. P. Van Duyne and L. D. Marks, J. Phys. Chem. C, 2013, 117, 15859-15870.

62 G. D. Barmparis, Z. Lodziana, N. Lopez and I. N. Remediakis, Beilstein J. Nanotechnol., 2015, 6, 361-368.

63 F. Silly and M. R. Castell, J. Phys. Chem. B, 2005, 109, 12316-12319.

64 X. Liu, M. H. Liu, Y. C. Luo, C. Y. Mou, S. D. Lin, H. Cheng, J. M. Chen, J. F. Lee and T. S. Lin, J. Am. Chem. Soc., 2012, 134, 10251-10258.

65 R. A. Bennett, C. L. Pang, N. Perkins, R. D. Smith, P. Morrall, R. I. Kvon and M. Bowker, J. Phys. Chem. B, 2002, 106, 4688-4696. 
66 R. Gubó, C. M. Yim, M. Allan, C. L. Pang, A. Berkó and G. Thornton, Top. Catal., 2018, 61, 308-317.

67 C. Wu, M. S. J. Marshall and M. R. Castell, J. Phys. Chem. C, 2011, 115, 8643-8652.

68 C. J. Johnson, E. Dujardin, S. A. Davis, C. J. Murphy and S. Mann, J. Mater. Chem., 2002, 12, 1765-1770.

69 Z. L. Wang, J. Phys. Chem. B, 2000, 104, 1153-1175.

70 L. Vitos, A. V. Ruban, H. L. Skriver and J. Kollár, Surf. Sci., 1998, 411, 186-202.

71 Y. Kuwauchi, H. Yoshida, T. Akita, M. Haruta and S. Takeda, Angew. Chemie Int. Ed., 2012, 51, 7729-7733.

72 N. Ta, J. Liu, S. Chenna, P. A. Crozier, Y. Li, A. Chen and W. Shen, J. Am. Chem. Soc., 2012, 134, 20585-20588.

73 W. Gao, A. S. Choi and J.-M. Zuo, Surf. Sci., 2014, 625, 16-22.

74 W. Gao, S. Sivaramakrishnan, J. Wen and J.-M. Zuo, Nano Lett., 2015, 15, 2548-2554.

75 T. Castro and R. Reifenberger, Phys. Rev. B, 1990, 42, 8548-8556.

76 G. Schmid and B. Corain, Eur. J. Inorg. Chem., 2003, 3081-3098. 